Relations industrielles

Industrial Relations

\title{
The Union Member Speaks. ROSEN, Hjalmar and R. A. HUDSON ROSEN, New York: Prentice-Hall, Inc., 1955, 250 pp.
}

\section{Roger Chartier}

Volume 11, numéro 2, mars 1956

URI : https://id.erudit.org/iderudit/1022651ar

DOI : https://doi.org/10.7202/1022651ar

Aller au sommaire du numéro

Éditeur(s)

Département des relations industrielles de l’Université Laval

ISSN

0034-379X (imprimé)

1703-8138 (numérique)

Découvrir la revue

Citer ce compte rendu

Chartier, R. (1956). Compte rendu de [The Union Member Speaks. ROSEN,

Hjalmar and R. A. HUDSON ROSEN, New York: Prentice-Hall, Inc., 1955, 250

pp.] Relations industrielles / Industrial Relations, 11(2), 122-123.

https://doi.org/10.7202/1022651ar

Tous droits réservés (C Département des relations industrielles de l’Université Laval, 1956
Ce document est protégé par la loi sur le droit d'auteur. L'utilisation des services d'Érudit (y compris la reproduction) est assujettie à sa politique d'utilisation que vous pouvez consulter en ligne.

https://apropos.erudit.org/fr/usagers/politique-dutilisation/ 


\section{LIVRES ET REVUES}

JEHRING, J.J., Profit-Sharing for Small Business, 1 broch. 53 pp. Profit Sharing Foundation, Evanston, 1955.

Cette brochure préparée pour le compte d'une institution cherchant à promouvoir la participation aux bénéfices n'a pas un caractère de propagande. L'auteur a voulu présenter aux dirigeants de petites entreprises, (cent employés et moins) qui sont déjà intéressés à ces plans des explications claires concernant les buts et modalités d'application. Il a tiré ses illustrations d'une enquête menée par la Fondation auprès de 78 petites entreprises ayant expérimenté un plan de participation soit immédiate ou différée. Ce n'est pas une étude scientifique, mais elle est très pratique pour quiconque veut avoir une idée de la participation aux bénéfices dans les petites entreprises.

G. D.

ORNATI, OscaR, Jobs and Workers in India, The Institute of International Industrial and Labor Relations, Cornell University, Ithaca, N.Y., I vol., 215 pp. Price, paper $\$ 3.00$, Cloth $\$ 4.00$.

L'Inde est dans le monde oriental un pays qui occupe une place stratégique. Par sa situation géographique, par sa population - qui comprend le sixième de celle du globe - par l'orientation de sa politique, elle est apelée à jouer un rôle très important dans l'expansion du communisme. Nous n'avons pas le droit de nous montrer indifférents à ce qui s'y passe. L'ouvrage du professeur ORNATI veut être une initiation à une partie des problèmes qui s'agitent aux Indes. Il a choisi de parler du monde du travail et spécialement du travail industriel. Dans quelques chapitres, il donne une vue panoramique de la population, de la main-d'oeuvre et de l'emploi, de l'avènement de l'industrie, du régime de travail et des salaires et de la législation du travail. Il fait aussi une brève histoire du mouvement ouvrier et décrit les groupements actuellement existants. Enfin il analyse les pratiques courantes dans l'aménagement des rela- tions du travail: négociations collectives, le règlement des différends industriels.

Avec une population de plus de 350 millions d'habitants, l'Inde n'a que 7 millions de travailleurs industriels. Cependant la production par tête de ces travailleurs dépasse grandement celle des autres catégories sociales. En outre, grâce à leurs organisations syndicales, ils ont dans leur pays une influence beaucoup plus considérable que ne l'indique leur chiffre numérique. Par contre, à cause de l'industrialisation assez récente, de la mobilité des travailleurs, du tempérament propre aux habitants du pays, et pour une grande part, à cause de l'incurie des employeurs à rénover leurs techniques de production et leurs conceptions des rapports humains dans l'entreprise, le monde ouvrier est inadapté à sa nouvelle condition. Tous employeurs et salariés ont tendance à s'en remettre à l'Etat pour règler leurs problèmes. Et cela se situe à l'intérieur d'une économie où, à la fois il y a une sous-production et un chômage grandissant.

Il était impossible pour l'auteur de traiter à fond tous les problèmes qu'il a abordés. Cependant son étude a le mérite de mettre entre les mains du lecteur une synthèse et d'attirer son attention sur la nécessité d'un réaménagement des ressources humaines de ce pays. Ce sera le travail de spécialistes. L'ouvrage contient un appendice très précieux dans lequel l'auteur a préparé une bibliographie des principaux volumes publiés sur l'Inde.

\section{GÉrard Dion}

ROSEN, HJalmar and R. A. Hudson Rosen, The Union Member Speaks. New York: Prentice-Hall, Inc., 1955, $250 \mathrm{pp}$.

Voici la plus récente des études fort patientes qui ont été menées, ces dernières années, sur les opinions des membres locaux. Ces études sont venues sur le tard, et se comptent presque sur les doigts des deux mains. Mentionnons celles du R.P. Theodore V. Purcell, s.j., The Worker Speaks His Mind on Company and Union (Cambridge: Harvard University Press, 1953); d'Arnold Rose, Union Solidarity (Minneapolis: University of Minnesota Press, 1952); de Leo- 
nard Sayles et George Strauss, The Local Union (New York: Harper \& Brothers, 1953); d'un groupe de professeurs de l'Université de l'Illinois, LaborManagement Relations in Illini City, 2 vols. (Champaign, Ill.: University of Illinois Institute of Labor and Industrial Relations, 1953); d'un groupe de chercheurs de l'Université de Chicago, certaines études fragmentaires qui font partie d'un projet plus vaste de recherche sur le travailleur américain, notamment: Joel Seidman, Jack London, and Bernard Karsh, Leadership in a Local Union, Why Workers Join Unions, et Political Consciousness in a Local Union; tous Reprints de l'Industrial Relations Center (University of Chicago), ainsi que quelques travaux miméographiés dirigés par John $\mathrm{P}$. Caldwell à l'Université de Chicago au cours des cinq dernières années, et quelques thèses, telle celle de Jack London présentée à l'Université de Chicago.

Le livre des Rosen, aux humbles proportions, s'efforce de nous livrer la pensée des travailleurs syndiqués sur leur syndicat, son rôle, ses chefs. Les auteurs examinent les attitudes ouvrières à l'endroit des procédures de négociation collective et de règlement des griefs, à l'égard de l'agent d'affaires, des assemblées syndicales et de l'action politique syndicale.

Il nous semble que les auteurs ne sont pas suffisamment parti des réponses obtenues, et n'ont pas réussi à systématiser avec clarté les nombreuses données accumulées grâce aux réponses fournies par des milliers de syndiqués à un questionnaire passablement élaboré.

Si le contenu du volume est quelque peu déficient, les aspects méthodologiques ont cependant reçu la part du lion, car les appendices se succèdent dans une bonne moitié du texte. C'est par là ciue le travail des Rosen est le plus intéressant: on trouve le texte du questionnaire, la façon et les résultats de l'étudepilote, la manière dont on a formulé le questionnaire et procédé à l'échantillonnage; on apprend comment tirer le meilleur parti possible du questionnaire envoyé par la poste et des nombreuses entrevues effectuées, et comment analyser statistiquement les relations qui existent entre les diverses opinions.

Un appareil méthodologique aussi compliqué, pour se justifier, doit donner des résultats plus élaborés, plus sûrs, moins 《impressionistic ». Le volume des Rosen reste encore à écrire, sur bien des points.

\section{Roger Chartier}

BLUMEN IsADORE, KOGAN MARVIN and McCARTHY PHILIP J.: The Industrial Mobility of labor as a probability process. Ithaca, 1955, Cornell University. Cornell studies in industrial and labor relations, No. VI, $163 \mathrm{pp}$.

Etude statistique dans laquelle les auteurs exposent la méthode d'analyse par probabilité utilisée dans la détermination de la mobilité des travailleurs d'une industrie à l'autre.

Etablissant un modèle simple de probabilité pour l'analyse du mouvement entre deux périodes voisines, ils développent par la suite des modèles complexes pour l'analyse de mouvements entre industries pour des points éloignés dans le temps et la prévision des travailleurs «inertes》 et «mobiles》 sur de longues périodes.

J. H. 